\title{
Maintaining group polarity
}

Collective cell migration is the movement of groups of adhering cells and occurs during normal embryonic morphogenesis and cancer metastasis. However, the way that cell migration coordination is regulated remains poorly understood. In Developmental Cell, Aman and Piotrowski report that both the Wnt- $\beta$-catenin signalling pathway and the fibroblast growth factor (Fgf) signalling pathway maintain the polarity of the zebrafish lateral line primordium during migration.

Researchers have recently begun to use the zebrafish lateral line, which consists of neuromast cells (mechanosensory organs), to study collective cell migration. A primordium migrates from head to tail to deposit groups of neuromast precursor cells at regular intervals. The authors found that lateral line development was impaired in adenomatous polyposis coli (apc) mutant embryos. After leaving behind disorganized bands of cells, the mutant primordia stalled and never reached the tail tip. Cells within the mutant primordium were motile but failed to undergo directed coordinated migration. APC is known to suppress Wnt- $\beta$-catenin signalling, and expression analysis of Wnt- $\beta$-catenin target genes showed that Wnt- $\beta$-catenin signalling extended to the whole primordium in apc mutants, whereas it is normally restricted to the leading zone. This suggests that primordia fail to migrate owing to the ectopic activation of Wnt- $\beta$-catenin signalling.

So how is polar Wnt- $\beta$-catenin signalling achieved? The authors show that polarity relies on the interaction between the Wnt$\beta$-catenin and Fgf signalling pathways. Fgf ligands are normally expressed in the primordium leading zone; however, they were expressed throughout $a p c$ mutant primordia, showing that

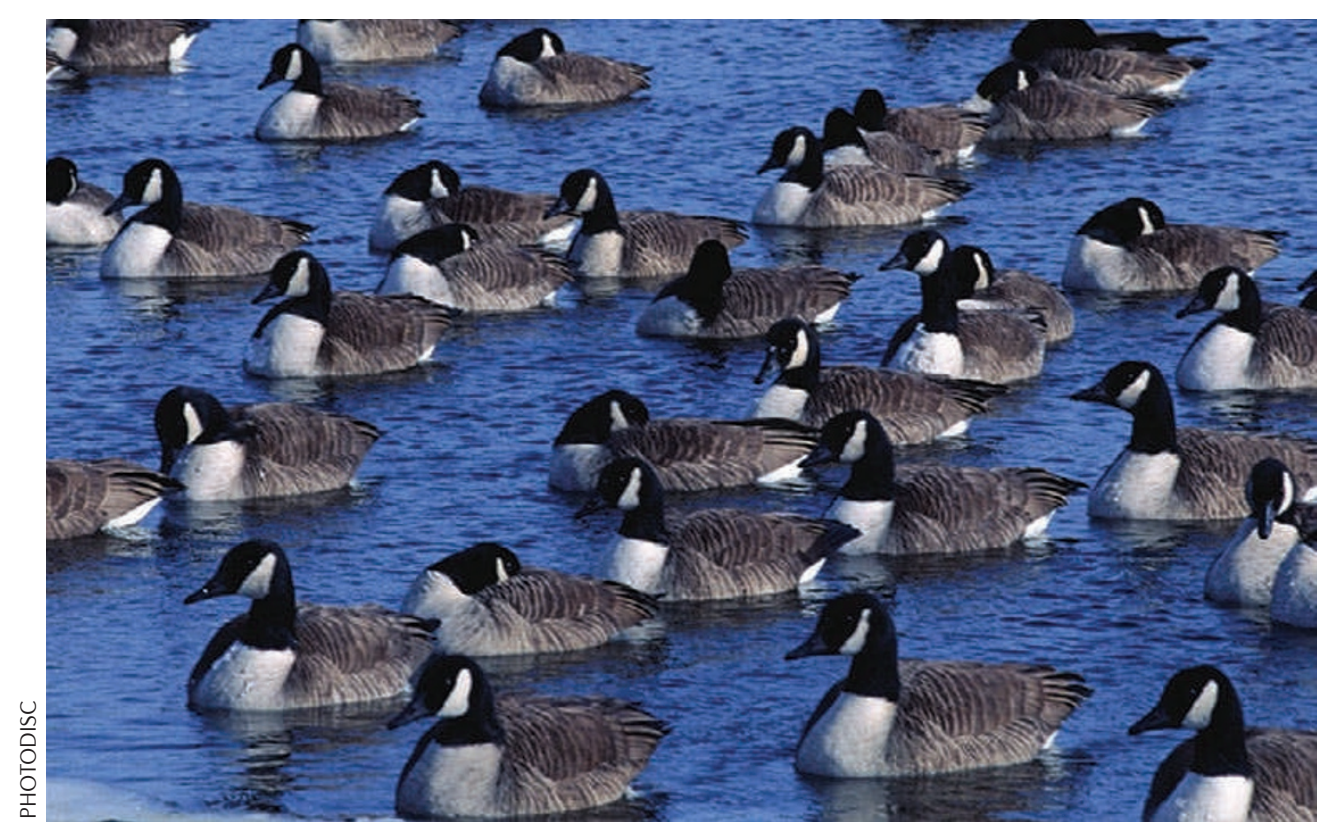

Wnt- $\beta$-catenin signalling induces Fgf ligand expression. Interestingly, Fgf signalling activity is restricted to the primordium-trailing zone, indicating that Fgf ligands act at a distance. Exclusion of Fgf signalling from the leading edge was due to a combination of low levels of Fgf receptor expression and the expression of an inhibitor (sef), which was also induced by the Wnt- $\beta$-catenin pathway. Furthermore, the authors found that the Fgf pathway blocks Wnt- $\beta$-catenin signalling at the rear by inducing expression of the Wnt- $\beta$-catenin pathway inhibitor dickkopf 1 .

Coordinated directional migration of the primordium also relies on the polarized expression of two chemokine receptors, $\mathrm{Cxcr} 4 \mathrm{~b}$ and $\mathrm{Cxcr} 7 \mathrm{~b}$, which are found at the front and rear of the primordium, respectively. Aman and Piotrowski show that this spatial restriction results from Wnt$\beta$-catenin expression at the leading edge inhibiting both $c x c r 7 b$ expression and a putative $c x c r 4 b$ repressor.

This study shows that the interaction between the Fgf and Wnt$\beta$-catenin pathways ensures their mutually exclusive activation, which in turn leads to the polarized distribution of chemokine receptors. Cxcr4 and Wnt signalling have important roles in the metastasis of several types of cancer. The authors suggest that elucidating signalling pathway interactions might provide further insight into how cancer cell clusters migrate.

Kim Baumann Editor

Cell Migration Gateway

ORIGINAL RESEARCH PAPER Aman, A. 8

Piotrowski, T. Wnt/ $\beta$-catenin and Fgf signaling control collective cell migration by restricting chemokine receptor expression. Dev. Cell 15, 749-761 (2008) 